\title{
Supplementation with omega fatty acids increases the mRNA expression level of PLA2G4A in patients with gastric cancer
}

\author{
Donya Bazhan ${ }^{1}$, Mahmoud Shekari Khaniani ${ }^{2}$ \\ ${ }^{1}$ Department of Cellular \& Molecular Biology, Islamic Azad University, Ahar Branch, Ahar, Iran; ${ }^{2}$ Department of Medical Genetics, Faculty of \\ Medicine, Tabriz University of Medical Sciences, Tabriz, Iran \\ Contributions: (I) Conception and design: None; (II) Administrative support: None; (III) Provision of study materials or patients: All authors; (IV) \\ Collection and assembly of data: All authors; (V) Data analysis and interpretation: All authors; (VI) Manuscript writing: All authors; (VII) Final \\ approval of manuscript: All authors. \\ Correspondence to: Mahmoud Shekari Khaniani, MD, PhD. Department of Medical Genetics, Faculty of Medicine, Tabriz University of Medical \\ Sciences, Tabriz, Iran. Email: mahmoud.khaniani@gmail.com.
}

Background: Many lines of evidence suggest that arachidonic acid (AA)-based eicosanoid signaling pathway involved in development and progression of human cancers. Cytosolic phospholipase A2- $\alpha$ (cPLA2 $\alpha$ ) encoded by the PLA2G4A gene acts as an upstream regulator of eicosanoid signaling pathway through providing intracellular AA. The current study aimed to evaluate the effect of omega fatty acids on mRNA expression level of PLA2G4A in patients with gastric cancer (GC) and to assess the possible relation between its expression and clinicopathological features.

Methods: According to treatment strategy, 34 chemotherapy-naive patients were randomly divided into two groups including, treatment group I (17 subjects received cisplatin alone) and treatment group II (17 individuals received cisplatin plus omega fatty acids) in a double-blind manner. The gastric biopsies specimens were taken from subjects before and after treatment and then mRNA expression level of PLA2G4A was evaluated by quantitative real-time PCR procedure.

Results: The expression of the PLA2G4A gene at the protein level in the gastric biopsies samples was also determined by immunohistochemistry. Our findings revealed a significantly up-regulated expression of PLA2G4A mRNA in treatment group II after receiving cisplatin plus omega fatty acid compared to before treatment $(\mathrm{P}=0.003)$. In treatment group $\mathrm{I}$, there was no significant difference in mRNA expression levels of PLA2G4A before and after treatment $(\mathrm{P}=0.790)$. We also found that mRNA expression of PLA2G4A in treatment group II was significantly associated with tumor size $(\mathrm{P}=0.007)$ and familial history $(\mathrm{P}=0.006)$.

Conclusions: This study provides evidence that supplementation with omega fatty acids increases the mRNA expression level of PLA2G4A in patients with GC and may be crucial in guarding the cell from transformation and carcinogenesis.

Keywords: Phospholipase A2; PLA2G4A; omega fatty acids; gastric cancer (GC)

Submitted Jun 05, 2018. Accepted for publication Jul 12, 2018.

doi: 10.21037/jgo.2018.08.12

View this article at: http://dx.doi.org/10.21037/jgo.2018.08.12

\section{Introduction}

Gastric cancer (GC) is the fourth commonly diagnosed malignancy in both sexes and is still the second leading cause of cancer-related mortality globally $(1,2)$. According to annual statistics reporting from the American Cancer
Society, 26,240 new cases of GC are expected to be diagnosed and 10,800 deaths will occur in the United State in 2018 (3). GC is a multifactorial disorder caused by the interplay of multiple genetic factors and other factors, such as lifestyle and environmental factors $(4,5)$. Several etiologic factors, including Helicobacter pylori bacterial infection, 
gastric polyps, mucosa-associated lymphoid tissue (MALT) lymphoma, obesity, high salt diets, ethnicity and smoking have already been identified for GC (6). However, many questions have been remained on the exact pathophysiologic mechanisms of GC.

It has been fairly well demonstrated that variation in endogenously synthesized eicosanoids level, which depends on the phospholipase enzymes activity may play a fundamental role in the development and progression of cancers $(7,8)$. In the humans, there are four major classes of phospholipases which catalyze the hydrolysis of membrane glycerophospholipids, termed phospholipase A1 (PLA1), phospholipase A2 (PLA2), phospholipase C (PLC) and phospholipase D (PLD) (9). The PLA2 enzymes (EC3.1.4.4) catalyze the hydrolysis of the acyl ester bond of membrane phospholipids at the sn-2 position to release biologically active fatty acids and lysophospholipids (10). The superfamily of PLA2 currently consists of five distinct types of enzymes: cytosolic PLA2 (cPLA2), calciumindependent PLA2s (iPLA2), secreted PLA2 (sPLA2), platelet-activating factor-acetylhydrolases PLA2 (PAFAHs) and lysosomal PLA2 (10). The cPLA2 family includes 4 subgroups in human: cPLA2 $\alpha$; PLA2 $\beta$; $\operatorname{cPLA} 2 \gamma$ and $\operatorname{cPLA} 2 \delta$ (11). The cPLA2 $\alpha$, which is encoded by the PLA2G4A gene, represents a marked preference for hydrolysis of the membrane phospholipids which contain arachidonic acid (AA) (12). The AA released from the phospholipids is metabolized by cyclooxygenase (COX) and lipoxygenase (LOX) enzyme-catalyzed pathways to produce eicosanoids such as prostaglandins (PG), thromboxanes (TX), hydroxyeicosatetraenoic acids (HETE) and leukotrienes (LT) (12). These AA-derived lipid mediators modulate a wide array of physiological processes, including cell proliferation, cell differentiation, apoptosis, cell motility, invasion and angiogenesis $(12,13)$. Not surprisingly, dysregulation of eicosanoid metabolism pathway component(s) expression, especially cPLA2 $\alpha$, contributes to initiation and progression of the various cancer types (14-16). Zhang et al. reported that mRNA expression level of PLA2G4A is decreased in patients with GC (14). However, the studies conducted by Sundarraj et al. and Patel et al. in non-small cell lung cancer and prostate cancer have shown conflicting results $(15,16)$. On the other hand, accumulating evidence during the past decades suggest that omega fatty acids exhibit substantial effects in preventing and treating of the malignancies (17-20). Hence, the current study was designed to determine the effect of omega fatty acids on mRNA expression level of PLA2G4A in chemotherapy-naive patients with GC. The possible relation between mRNA expression level of PLA2G4A and clinicopathological parameters was also evaluated.

\section{Methods}

\section{Participants}

The biopsies samples (before and after treatment) from 34 chemotherapy-naive patients with histologically and cytologically confirmed GC were collected at the Liver and Gastrointestinal Diseases Research Center (LGDRC) and Hematology-Oncology Research Center, Tabriz, Iran, during 2013-2015. All obtained gastric biopsy specimens from patients were stored in RNAlater solution (Qiagen, Germany) at $-80{ }^{\circ} \mathrm{C}$ until RNA extraction. According to treatment strategy, subjects were divided into two groups including treatment group I (17 individuals; mean age, $67.5 \pm 11.21$ years) received daily cisplatin $75 \mathrm{mg} / \mathrm{m}^{2}$ alone and treatment group II (17 individuals; mean age, $71.25 \pm 9.81$ years) received daily cisplatin $75 \mathrm{mg} / \mathrm{m}^{2}$ plus orally administered omega fatty acid supplements $3,600 \mathrm{mg}$ per day for three-course. Patients with the following criteria were excluded from the study: cardiac obstruction, pyloric obstruction, diabetes, renal disorders, inflammatory diseases and individuals who were used omega fatty acids supplement for three months before sampling. The histological grade and tumor size of all obtained biopsy samples were determined by histopathological analysis. A structured questionnaire form was used to obtain demographic data from all participants and these data are shown in Table 1. This study was approved by the Ethics and Human Rights Committee of Tabriz University of Medical Sciences (ethical code number 92213) and written informed consent was obtained from all subjects after the purpose of study was explained.

\section{RNA extraction and cDNA Synthesis}

Total RNA from gastric biopsies samples was extracted using the total RNA extraction RNeasy kit (Qiagen, Valencia, CA) and treatment of isolated RNA with RNasefree DNase (MBI Fermentas, Canada) was also performed based on the manufacturer's instructions. The quantity and integrity of purified RNA were assessed photometrically using NanoDrop ${ }^{\circledR}$ ND-1000 Spectrophotometer (Thermo Scientific, USA) and observation of $28 \mathrm{~S}$ and $18 \mathrm{~S}$ ribosomal 
Table 1 Demographic and clinical properties of two treatment groups

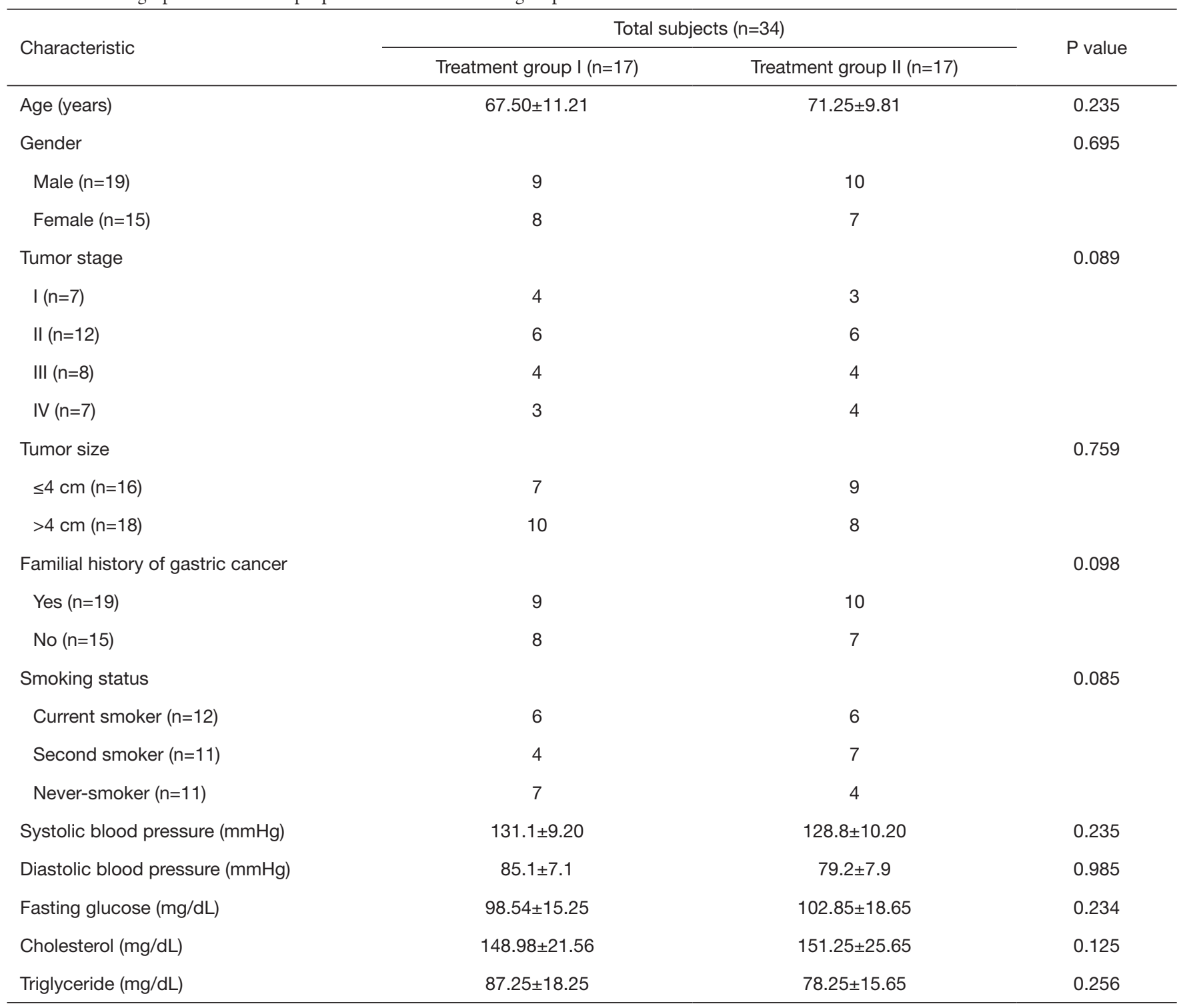

RNA bands after electrophoresis on $0.8 \%$ agarose gel, respectively. Finally, complementary DNA (cDNA) was synthesized from $1 \mu \mathrm{g}$ of DNase I-treated total RNA using RevertAid $^{\mathrm{TM}}$ First Strand cDNA Synthesis Kit (Fermentas, Canada) with random hexamer primer following the manufacturer's protocol and published method (21).

\section{Quantitative real-time PCR}

To evaluate the mRNA expression level of PLA2G4A in 34 patients diagnosed with GC (before and after treatment), we used quantitative real-time PCR (qPCR) procedure. The qPCR was performed in triplicate by a Rotor-Gene ${ }^{\mathrm{TM}} 6000$ system (Corbett Research; Mortlake, New South Wales, Australia) using genespecific primers and SYBR Green Master Mix (SYBR ${ }^{\circledR}$ Premix Ex Taq ${ }^{\mathrm{TM}}$ ) (TaKaRa, Japan) following the manufacturer's recommendations. The primer pairs for PLA2G4A F 5'-GACGTGCTGGGAAGGTACAC-3' and R 5'-AGCCCACTGTCCACTACA-3' and for GAPDH F 5'-GAAGGTGAAGGTCGGAGTC-3' and R 5'-GAAGATGGTGATGGGATTTC-3' were used in the amplification assays. The amplification reactions were carried out in a final volume of $25 \mu \mathrm{L}$ with the following 
components: $100 \mathrm{ng}$ cDNA, SYBR Green Master Mix 2×, ROX dye $50 \times, 10$ pmol of forward and reverse primers for PLA2G4A and GAPDH genes. Thermocycling conditions were as follows: $94{ }^{\circ} \mathrm{C}$ for $5 \mathrm{~min}, 50$ cycles (for PLA2G4A) and 35 cycles (for GAPDH) of $94{ }^{\circ} \mathrm{C}$ for $10 \mathrm{~s}$, annealing at $60{ }^{\circ} \mathrm{C}$ (for PLA2G4A) and $61{ }^{\circ} \mathrm{C}$ (for GAPDH) for $15 \mathrm{~s}$, and extension at $72{ }^{\circ} \mathrm{C}$ for $20 \mathrm{~s}$. Efficiency of qPCR was evaluated using serially diluted cDNA method and standard curves were plotted in each run. The mRNA expression level of PLA2G4A in the biopsy samples were normalized using the mean expression of the house keeping gene GAPDH. The relative fold change in the gene expression of PLA2G4A was determined by the $2^{-\Delta \Delta \mathrm{Ct}}$ comparative method. We also used no template sample as a negative control.

\section{Immunobistochemistry}

The cPLA2 $\alpha$ protein expression on $5 \mu \mathrm{m}$ sections of biopsy samples was determined by immunohistochemistry (IHC) technique. The sections of biopsy samples were incubated with a monoclonal cPLA2 $\alpha$ antibody that can be visualized through staining.

\section{Statistical analysis}

The distribution of mRNA expression levels of PLA2G4A around cutoff points were analyzed using KolmogorovSmirnov test. This analysis revealed that data were not normally distributed and therefore non-parametric statistical tests were used to compare the data. We used two-tailed Mann-Whitney U test to evaluate the statistically significant difference in mRNA expression levels of PLA2G4A before and after treatment in both groups. The possible relation between clinicopathological parameters and the mRNA expression levels of PLA2G4A was examined using one way ANOVA test. The correlation between mRNA expression level of PLA2G4A and clinicopathological features in both groups was analyzed by Pearson's correlation coefficient test. All statistical analysis was carried out using SPSS version 16.0 statistic software package (SPSS Inc., Chicago, IL, USA), and all continuous variables were expressed as mean \pm standard deviation (SD). $\mathrm{P}$ values less than 0.05 were statistically considered as significant.

\section{Results}

The mRNA expression level of PLA2G4A in 34 patients with GC before and after treatment was quantified by qPCR method. Based on initial analysis of data using Kolmogorov-Smirnov test the mean of PLA2G4A mRNA expression level was chosen as a cut-off value. As shown in Figure 1, the mRNA expression level of PLA2G4A was significantly higher in treatment group II after treatment with cisplatin plus omega fatty acids, compared to before treatment $(\mathrm{P}=0.003)$ (Figure 1). No statistical difference was observed on mRNA expression level of PLA2G4A between before and after receiving cisplatin alone in group $\mathrm{I}(\mathrm{P}=0.790)$ (Figure 1$)$. The cPLA2 $\alpha$ protein expression was also examined in sections of biopsy samples using IHC method. We found that PLA2G4A gene was expressed at protein level in gastric biopsies samples.

Analysis of the possible association between the mRNA expression levels of PLA2G4A and clinicopathological parameters was performed separately for both groups (Table 2). Our results showed that the mRNA expression levels of PLA2G4A were not significantly related to sex, histological stage, tumor size and family history of cancer in treatment group I ( $\mathrm{P}>0.05)$ (Table 2). In treatment group II, higher levels of PLA2G4A mRNA was associated with sex $(\mathrm{P}=0.009)$, tumor size $(\mathrm{P}=0.007)$ and family history of cancer $(\mathrm{P}=0.006)$ (Table 2). No association was found between mRNA expression level of PLA2G4A and histological stage in treatment group II ( $>>0.05)$ (Table 2).

The correlation between the relative expression of PLA2G4A and clinicopathological parameters was analyzed by Pearson's correlation test (Table 3). The results showed that relative expression level of PLA2G4A in treatment group I were not significant correlated with age, sex, histological stage, tumor size and family history of cancer $(\mathrm{P}>0.05)$ (Table 3). However, we found that relative expression of PLA2G4A in treatment group II was significantly and positively correlated with histological stage $(\mathrm{P}=0.038)$ and tumor size $(\mathrm{P}=0.022)$ (Table 3).

\section{Discussion}

Over the past decades, results of cell culture, animal models, and epidemiological and clinical studies have provided evidence to support the anti-carcinogenic effects of omega fatty acids (18-20,22,23). Although the precise molecular mechanisms by which omega fatty acids inhibit the development and progression of cancers has not understood very well, several possible explanations have been suggested by earlier studies. Sun et al. previously demonstrated that the omega fatty acids can induce apoptosis in the human 


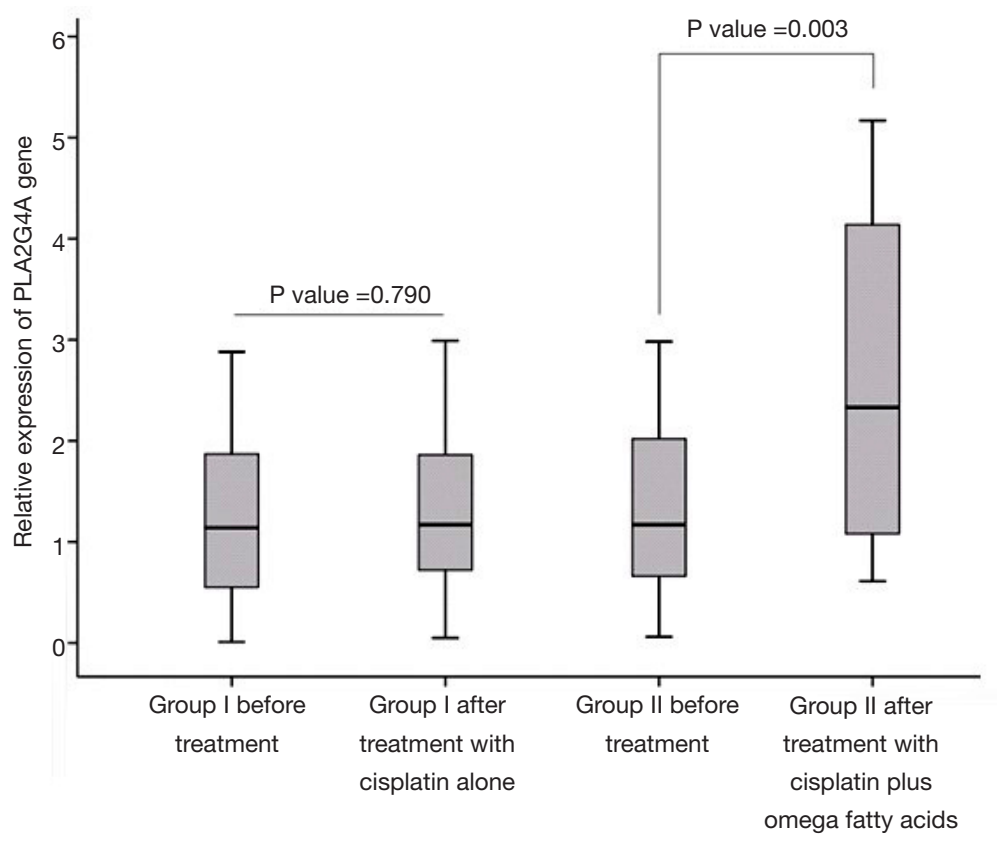

Figure 1 Box plot graph depicting the mRNA expression level of PLA2G4A in two treatment groups before and after treatment.

Table 2 mRNA expression level of PLA2G4A in relation with clinicopathological parameters

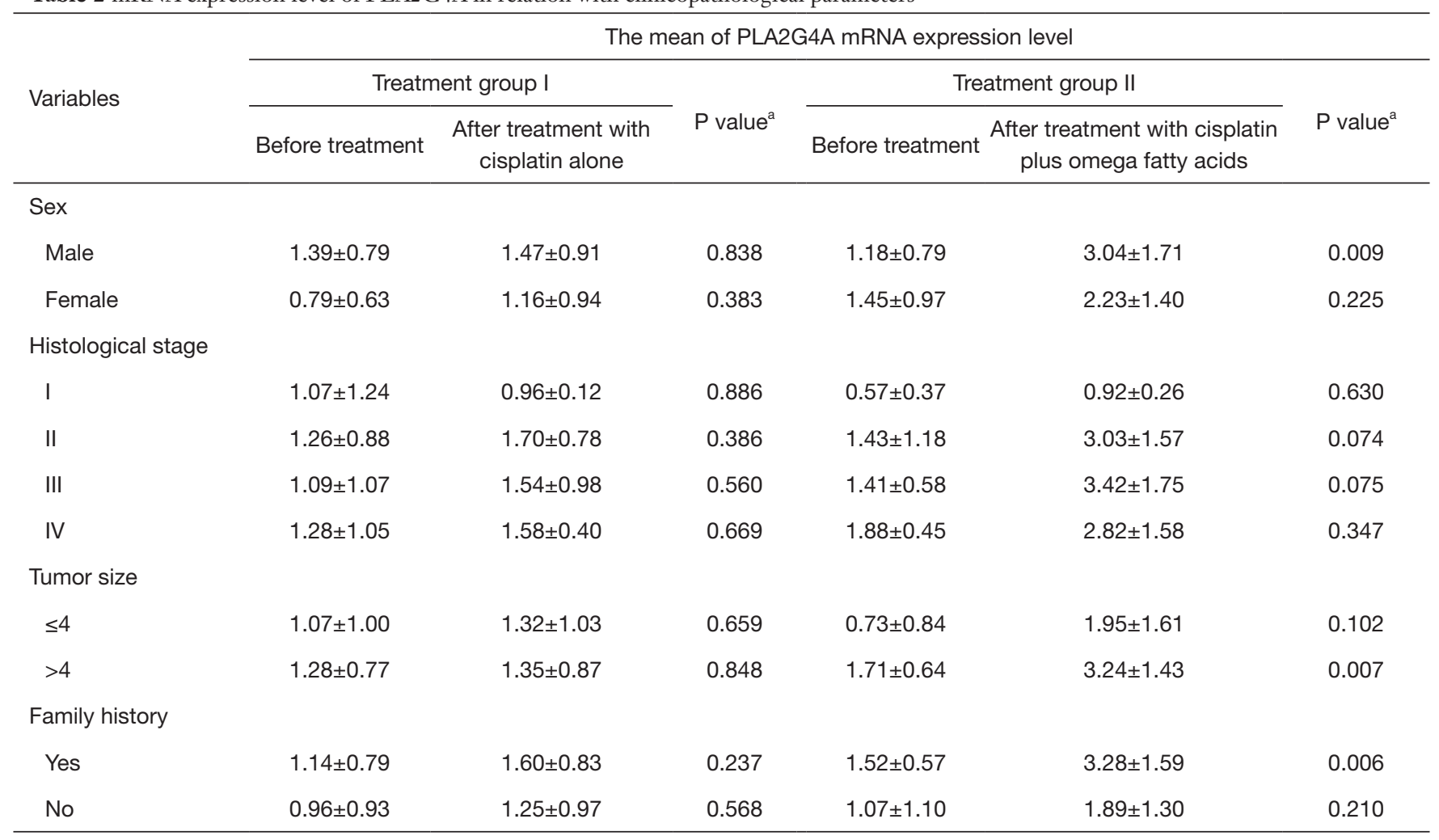

${ }^{\text {a }}$, P value calculated by Student's $t$-test. 
Table 3 Correlation between PLA2G4A mRNA expression levels with clinicopathological parameters

\begin{tabular}{|c|c|c|}
\hline \multirow{2}{*}{ Variables } & \multicolumn{2}{|c|}{ PLA2G4A mRNA expression levels } \\
\hline & Treatment group I & Treatment group II \\
\hline \multicolumn{3}{|l|}{ Age } \\
\hline$r^{*}$ & 0.008 & 0.225 \\
\hline$P$ value & 0.963 & 0.201 \\
\hline \multicolumn{3}{|l|}{ Sex } \\
\hline$r^{\star}$ & 0.314 & 0.120 \\
\hline$P$ value & 0.071 & 0.497 \\
\hline \multicolumn{3}{|c|}{ Histological stage } \\
\hline$r^{\star}$ & 0.188 & 0.357 \\
\hline$P$ value & 0.287 & $0.038^{*}$ \\
\hline \multicolumn{3}{|c|}{ Tumor size } \\
\hline$r^{*}$ & 0.072 & 0.392 \\
\hline$P$ value & 0.687 & $0.022^{*}$ \\
\hline \multicolumn{3}{|c|}{ Family history } \\
\hline$r^{\star}$ & 0.153 & 0.283 \\
\hline$P$ value & 0.387 & 0.105 \\
\hline
\end{tabular}

*, values represent Pearson's correlation coefficient.

breast cancer cell line MCF-7 through inhibition of MEK/ Erk/Bad signaling pathway (24). In a similar study, Kang and co-workers reported the effect of docosahexaenoic acid (DHA) in induction of apoptotic cell death in human MCF-7 breast cancer cells via caspase 8 activation (25). Down-regulation of the $\mathrm{Wnt} / \beta$-catenin signaling pathway is one of the mechanisms through which omega fatty acids exerts their anti-proliferative activity in human pancreatic cancer cells (SW1990 and PANC-1) (26). Incubation of the human breast cancer cell line MDA-MB-231 with omega fatty acids leads to decrease cell proliferation possibly by decreasing signal transduction through the Akt/NFkB cell survival pathway (27). A recent study demonstrated that the cell cycle arrest at G0/G1 phase and the down-regulation of the CDK2 and cyclin E expression triggered by omega fatty acids in human neuroblastoma LAN-1 cells (28). A study conducted by Abdi et al., revealed that supplementation or combination therapy with omega fatty acids in multiple myeloma (MM) chemotherapy could increase drug sensitivity to bortezomib and reduce chemoresistance (29). Furthermore, mRNA expression analysis results pointed out that omega fatty acids modulate gene expression of proteins involved in Delta-Notch, Hedgehog (HHt) and Wnt signaling pathways (29). Treatment of PA-1, H1299, D54MG and SiHa cancer cell lines with omega fatty acids reduce cell viability and induce apoptosis which is mediated by mitogen-activated protein kinase (MAPK) activation (30). The potential effect of omega fatty acids supplementation in treatment of lung cancer has been investigated by Kikawa et al. (31). They reported that omega fatty acids can inhibit tumor cell growth by increasing oxidative stress in the A549 lung adenocarcinoma cell line (31). Alterations in fluidity, structure and/or function of lipid rafts have substantial consequences on plasma membrane organization and functionalities such as signal transduction, cell growth and migration $(32,33)$. Because wide arrays of membranesignaling proteins are located within the lipid rafts, change in lipid composition of membrane rafts can alter activity of these signaling proteins (32). The effects of omega fatty acids on the lipid composition of lipid rafts and epidermal growth factor receptor (EGFR) raft localization in MDAMB-231 human breast cancer cells has been previously studied (34). Omega fatty acids may exert their growth inhibitory effects on cancer cells by modifying the lipid composition of membrane rafts and EGFR signaling (34). Similarly, Corsetto et al. documented that omega fatty acids reduce the cell proliferation in MCF-7 and MDAMB-231 breast cancer cell lines probably through change the biophysical properties of lipid rafts decreasing the content of cholesterol and probably the distribution of key proteins (35). Hence, omega fatty acids, as precursors for the endogenous biosynthesis of bioactive lipid mediators, have been considered as potential chemopreventive and chemotherapeutic agents in treatment of malignancies. Meanwhile, the cPLA2 $\alpha$ enzyme, as an important upstream regulator, clearly plays a key role in production of these lipid mediators. Therefore, we investigated the effect of omega fatty acids on mRNA expression level of PLA2G4A in patients with GC. It is worth noting that the current study is the first report evaluating the effect of omega fatty acids on mRNA expression level of PLA2G4A in patients with GC, to the authors' knowledge. We found that the expression of PLA2G4A at mRNA level is significantly increased in treatment group II after receiving cisplatin plus omega fatty acid compared to before treatment $(\mathrm{P}<0.05)$ (Figure 1). A low mean expression level of PLA2G4A gene was observed in patients before supplementation with omega fatty acids (Figure 1). These data are consistent with other studies $(14,36)$. A significantly reduced expression of 
PLA2G4A in GC has been reported by Zhang et al. (14). The results of this study suggest that the reduced expression of PLA2G4A is associated with unfavorable outcome for patients with GC (14). A study using immunohistochemical technique clearly revealed that the expression of PLA2G4A at protein level is markedly decreased in human colorectal tumors (36). Homozygous and heterozygous deletion of cPLA2 increases the azoxymethane-induced colon carcinogenesis in mice (37). It therefore seems likely that down-regulated expression of PLA2G4A may contribute to transformation and tumor progression. On the contrary, over expression of PLA2G4A has been reported in prostate cancer (16), non-small cell lung cancer (15), and squamous cell carcinomas (38). No reasons were given for these contradictories. It is argued that cPLA2 $\alpha$ probably plays a dual role in carcinogenesis, depending on the tumor type.

In conclusion, our results suggested that omega fatty acids supplementation up-regulate the mRNA expression level of PLA2G4A in patients with GC. These data provide additional evidence to support the hypothesis that omega fatty acids, as potent antineoplastic agents, may improve the outcomes of chemotherapy. However, further studies are necessary to disclose the exact molecular mechanisms by which omega fatty acids regulate PLA2G4A gene expression.

\section{Acknowledgements}

The authors would like to express sincerest appreciation to Dr. Mohammad Hossein Somi for his cooperation in collecting samples and Dr. Ali Esfahani, Dr. Homayun Dolatkhah and Mr. Asghar Hosseinzadeh for their unreserved help throughout this project. We are truly thankful to Hossein Samadi Kafil for his great help during the molecular laboratory works. Also, we would like to extend our gratitude to all subjects for participating in the current study.

\section{Footnote}

Conflicts of Interest: The authors have no conflicts of interest to declare.

Ethical Statement: The current study was approved by the Ethics and Human Rights Committee of Tabriz University of Medical Sciences (No. 92213) and informed consent was taken from all the patients.

\section{References}

1. Rahman R, Asombang AW, Ibdah JA. Characteristics of gastric cancer in Asia. World J Gastroenterol 2014;20:4483-90.

2. Alimoglu O, Sisik A, Eren T, et al. Analysis of surgically treated gastric cancers: a tertiary hospital experience in Turkey. North Clin Istanb 2015;2:101-6.

3. Siegel RL, Miller KD, Jemal A. Cancer statistics, 2018. CA Cancer J Clin 2018;68:7-30.

4. Zabaleta J. Multifactorial etiology of gastric cancer. Methods Mol Biol 2012;863:411-35.

5. Goral V. Etiopathogenesis of Gastric Cancer. Asian Pac J Cancer Prev 2016;17:2745-50.

6. Karimi P, Islami F, Anandasabapathy S, et al. Gastric cancer: descriptive epidemiology, risk factors, screening, and prevention. Cancer Epidemiol Biomarkers Prev 2014;23:700-13.

7. Cathcart MC, Lysaght J, Pidgeon GP. Eicosanoid signalling pathways in the development and progression of colorectal cancer: novel approaches for prevention/ intervention. Cancer Metastasis Rev 2011;30:363-85.

8. Lenihan-Geels G, Bishop KS, Ferguson LR. Cancer Risk and Eicosanoid Production: Interaction between the Protective Effect of Long ChainOmega- Polyunsaturated Fatty Acid Intake and Genotype. J Clin Med 2016;5:E25.

9. Aloulou A, Ali YB, Bezzine S, et al. Phospholipases: an overview. Methods Mol Biol 2012;861:63-85.

10. Burke JE, Dennis EA. Phospholipase A2 biochemistry. Cardiovasc Drugs Ther 2009;23:49-59.

11. Sun GY, Shelat PB, Jensen MB, et al. Phospholipases A2 and inflammatory responses in the central nervous system. Neuromolecular Med 2010;12:133-48.

12. Wang D, Dubois RN. Eicosanoids and cancer. Nat Rev Cancer 2010;10:181-93.

13. Scott KF, Sajinovic M, Hein J, et al. Emerging roles for phospholipase A2 enzymes in cancer. Biochimie 2010;92:601-10.

14. Zhang X, Wu Q, Gan L, et al. Reduced group IVA phospholipase A2 expression is associated with unfavorable outcome for patients with gastric cancer. Med Oncol 2013;30:454.

15. Sundarraj S, Kannan S. Immunohistochemical expression of cytosolic phospholipase A2a in non-small cell lung carcinoma. Asian Pac J Cancer Prev 2010;11:1367-72.

16. Patel MI, Singh J, Niknami M, et al. Cytosolic phospholipase A2-alpha: a potential therapeutic target for prostate cancer. Clin Cancer Res 2008;14:8070-9. 
17. Serini S, Piccioni E, Merendino N, et al. Dietary polyunsaturated fatty acids as inducers of apoptosis: implications for cancer. Apoptosis 2009;14:135-52.

18. Jing K, Wu T, Lim K. Omega-3 polyunsaturated fatty acids and cancer. Anticancer Agents Med Chem 2013;13:1162-77.

19. Berquin IM, Edwards IJ, Chen YQ. Multi-targeted therapy of cancer by omega-3 fatty acids. Cancer Lett 2008;269:363-77.

20. Gu Z, Shan K, Chen H, et al. n-3 Polyunsaturated Fatty Acids and their Role in Cancer Chemoprevention. Curr Pharmacol Rep 2015;1:283-94.

21. Hu R, Buck NE, Khaniani MS, et al. Gene induction for the treatment of methylmalonic aciduria. J Gene Med 2009;11:361-9.

22. Cockbain AJ, Toogood GJ, Hull MA. Omega-3 polyunsaturated fatty acids for the treatment and prevention of colorectal cancer. Gut 2012;61:135-49.

23. Eltweri AM, Thomas AL, Metcalfe M, et al. Potential applications of fish oils rich in omega-3 polyunsaturated fatty acids in the management of gastrointestinal cancer. Clin Nutr 2017;36:65-78.

24. Sun H, Hu Y, Gu Z, et al. Omega-3 fatty acids induce apoptosis in human breast cancer cells and mouse mammary tissue through syndecan-1 inhibition of the MEK-Erk pathway. Carcinogenesis 2011;32:1518-24.

25. Kang KS, Wang P, Yamabe N, et al. Docosahexaenoic acid induces apoptosis in MCF-7 cells in vitro and in vivo via reactive oxygenspecies formation and caspase 8 activation. PLoS One. 2010;5:e10296.

26. Song KS, Jing K, Kim JS, et al. Omega-3-polyunsaturated fatty acids suppress pancreatic cancer cell growth in vitro and in vivo via downregulation of $\mathrm{Wnt} /$ Beta-catenin signaling. Pancreatology 2011;11:574-84.

27. Schley PD, Jijon HB, Robinson LE, et al. Mechanisms of omega-3 fatty acid-induced growth inhibition in MDAMB-231 human breast cancer cells. Breast Cancer Res Treat 2005;92:187-95.

28. So WW, Liu WN, Leung KN. Omega-3 Polyunsaturated Fatty Acids Trigger Cell Cycle Arrest and Induce

Cite this article as: Bazhan D, Khaniani MS. Supplementation with omega fatty acids increases the mRNA expression level of PLA2G4A in patients with gastric cancer. J Gastrointest Oncol 2018;9(6):1176-1183. doi: 10.21037/jgo.2018.08.12
Apoptosis in Human Neuroblastoma LA-N-1 Cells. Nutrients 2015;7:6956-73.

29. Abdi J, Garssen J, Faber J, et al. Omega-3 fatty acids, EPA and DHA induce apoptosis and enhance drug sensitivity in multiplemyeloma cells but not in normal peripheral mononuclear cells. J Nutr Biochem 2014;25:1254-62.

30. Jeong S, Jing K, Kim N, et al. Docosahexaenoic acidinduced apoptosis is mediated by activation of mitogenactivated proteinkinases in human cancer cells. BMC Cancer 2014;14:481.

31. Kikawa KD, Herrick JS, Tateo RE, et al. Induced oxidative stress and cell death in the A549 lung adenocarcinoma cell line by ionizingradiation is enhanced by supplementation with docosahexaenoic acid. Nutr Cancer 2010;62:1017-24.

32. Siddiqui RA, Harvey KA, Zaloga GP, et al. Modulation of lipid rafts by Omega-3 fatty acids in inflammation and cancer: implications for use of lipids during nutrition support. Nutr Clin Pract 2007;22:74-88.

33. Zalba S, Ten Hagen TL. Cell membrane modulation as adjuvant in cancer therapy. Cancer Treat Rev 2017;52:48-57.

34. Schley PD, Brindley DN, Field CJ. (n-3) PUFA alter raft lipid composition and decrease epidermal growth factor receptor levels in lipid rafts of human breast cancer cells. J Nutr 2007;137:548-53.

35. Corsetto PA, Cremona A, Montorfano G, et al. Chemicalphysical changes in cell membrane microdomains of breast cancer cells after omega-3 PUFA incorporation. Cell Biochem Biophys 2012;64:45-59.

36. Dong M, Johnson M, Rezaie A, et al. Cytoplasmic phospholipase A2 levels correlate with apoptosis in human colon tumorigenesis. Clin Cancer Res 2005;11:2265-71.

37. Ilsley JN, Nakanishi M, Flynn C, et al. Cytoplasmic phospholipase A2 deletion enhances colon tumorigenesis. Cancer Res 2005;65:2636-43.

38. Zhang S, Du Y, Tao J, et al. Expression of cytosolic phospholipase A2 and cyclooxygenase 2 and their significance in humanoral mucosae, dysplasias and squamous cell carcinomas. ORL J Otorhinolaryngol Relat Spec 2008;70:242-8. 